\title{
Caracterización de la Tenencia de Animales de Compañía en la Ciudad de Buenos Aires, Argentina
}

\author{
Characterization of Pet Ownership in Buenos Aires City, Argentina \\ Analía Tortosa ${ }^{1,2}$, Romina Zumpano ${ }^{1}$, Inés Ardiles ${ }^{2}$, Yanina Berra ${ }^{1}$, \\ Adriana N. Faigenbaum ${ }^{2}$, Guillermo G. Guido ${ }^{2}$, Jorge Castro ${ }^{2}$, José L. Molina ${ }^{1,2}$, \\ Edgardo R. Marcos ${ }^{1,2}$, Osvaldo J. Degregorio ${ }^{1,3}$
}

\section{Resumen}

\begin{abstract}
El objetivo del estudio fue conocer características demográficas y de la tenencia de animales de compañía, así como las motivaciones, actitudes y conductas de la población que tiene o no animales, especialmente caninos, en la ciudad de Buenos Aires. Se diseñó un estudio social explicativo de prevalencia en dos áreas de la ciudad con diferentes estructuras socioeconómicas. En el grupo 1 (San Cristóbal) se encuestaron 357 viviendas y en el grupo 2 (Barrio Mitre) se encuestaron 169 viviendas. Se observó una alta proporción de animales por vivienda en ambas áreas. Los índices de tenencia de caninos por habitante entre las áreas difieren significativamente, lo que puede atribuirse al nivel socioeconómico. Las diferencias en las proporciones de caninos hembra, de talla pequeña y con tendencia a razas puras con indicadores de mortalidad, natalidad y supervivencia variables indicarían que las poblaciones de animales de compañía tienden a ser reflejo de las condiciones sociales y vida de la población humana. Se observó que la esterilización puede emplearse para el control reproductivo o para resolver necesidades de la estrecha convivencia en el área urbana. Mayoritariamente, los caninos son incorporados a través del regalo, la compra o la recolección en la vía pública. La adopción no fue considerada como opción para obtener un canino. Los mapas cognitivos de tenencia de caninos para caracterizar cualitativa y cuantitativamente el vínculo entre animales y sus tenedores demostraron ser una novedosa forma de analizar la interrelación de variables.
\end{abstract}

Palabras clave: tenencia, animales de compañía, motivaciones, conductas

\footnotetext{
${ }^{1}$ Cátedra Salud Pública, Facultad Ciencias Veterinarias, Universidad de Buenos Aires, Argentina

${ }^{2}$ Instituto de Zoonosis Dr. Luis Pasteur, Ciudad Autónoma de Buenos Aires, Argentina

${ }^{3}$ E-mail: odegre@fvet.uba.ar
}

Recibido: 30 de diciembre de 2015

Aceptado para publicación: 12 de abril de 2016 
The aim of this study was to determine demographic and ownership pet characteristics, motivations, attitudes and behaviours of the human population raising or not raising pets, especially dogs in Buenos Aires city. A prevalence explanatory social study was designed in two areas of the city with different socio-economic structure. A survey was conducted in 357 households in San Cristóbal (group 1) and in 169 households in Barrio Mitre (group 2). A high proportion of animals per household in both areas was observed. Per capita canine ownership rates significantly differed which can be attributed to different socio-economic status. Differences in the proportion of canine female, small size and purebred with variation in mortality, birth and survival rates indicate that pet populations tend to be reflection of social and living conditions of the human population. It was observed that sterilization can be used for reproductive control or to meet needs of close coexistence in urban areas. Mostly, canines are incorporated through gift, purchase or collection in public places. Adoption was not considered an option to get a dog. Cognitive maps of canine ownership were performed to characterize qualitatively and quantitatively the link between animals and their holders and proved to be a novel way to analyse the interrelationship of variables.

Key words: possession, pet, motivations, behaviours

\section{INTRODUCCIÓN}

Los animales que conviven con las poblaciones humanas en el medio urbano se han convertido en un significativo componente del bienestar del hombre con implicancias económicas, afectivas y sociales (Franco et al., 1989; Bennett y Rohlf, 2007).

La estructura y los cambios en las poblaciones de caninos y felinos dependen de un gran número de factores. Son especies con un potencial reproductivo que les permitiría un crecimiento exponencial. Varían en sus índices de natalidad y mortalidad de tal forma que puede darse un equilibrio, crecimiento o decrecimiento (Teclaw et al., 1992; Alcock, 2003).

Cada hábitat tiene una capacidad específica para cada especie y esta depende de la disponibilidad, distribución y calidad de recursos. En el caso de las poblaciones de animales de compañía en áreas urbanas, la capacidad del hábitat se encuentra influida por las decisiones del hombre, entrelazándose aspectos biológicos con factores relacionados con la satisfacción de necesidades y la influencia de las demandas sociales (Acosta et al., 2010). Las actitudes son el reflejo de los valores sociales del grupo y de los patrones conductuales propios de los miembros de ese grupo y sus interacciones, es decir, la conducta social (Blachshaw y Day, 1994; Bennett y Rohlf, 2007).

En los últimos años se han incrementado los estudios con técnicas que permiten la medición de actitudes de las personas que influyen en las conductas de los animales (Barberá Heredia, 1999; Adams et al., 2000). Así, se han evaluado aspectos relacionados con la interacción de animales y personas en espacios y el conocimiento de las personas sobre las enfermedades que pueden transmitirse al hombre y la influencia del ambientesocial. También se han analizado las percepciones que tienen expertos en ciencias veterinarias en relación con las motivaciones, actitudes y conductas de la población que tiene animales de compañía (Degregorio et al., 2005, 2010). 
Mapas cognitivos orientados hacia las conductas sociales han sido empleados para identificar características que permitan analizar actitudes, motivaciones y conductas que permitan predecir las decisiones en la tenencia de animales y analizar sus relaciones (Ozesmi U y Ozesmi SL, 2004; Degregorio et al., 2007, 2010).

El objetivo planteado en el presente estudio fue conocer las características demográficas y de la tenencia, así como las motivaciones, actitudes y conductas de la población que tiene o no animales, especialmente caninos, en la ciudad de Buenos Aires.

\section{Materiales y Métodos}

Se diseñó un estudio social explicativo de prevalencia para conocer características demográficas de las poblaciones de personas y caninos y de la tenencia de estos últimos en dos áreas de la ciudad de Buenos Aires en el año 2013. Se realizó un muestreo por etapas considerando primeramente las características socioeconómicas (Gobierno Ciudad de Buenos Aires, 2005a,b) y en una segunda etapa un muestreo aleatorio simple sin reposición en cada área seleccionada.

Se estudiaron dos áreas: San Cristóbal, considerado como nivel socioeconómico medio, con 144 manzanas y 18792 viviendas, y Barrio Mitre, considerado como nivel socioeconómico bajo, con 16 manzanas y 324 viviendas (INDEC, 2001).

El número mínimo de encuestas a realizar en viviendas fue estimado en 352 en el grupo 1 (San Cristóbal) y 160 en el grupo 2 (Barrio Mitre). Se consideró una distribución normal estandarizada $(\mathrm{z}=1.96)$, una confianza de $95 \%(1-\alpha=0.05)$, una precisión de $80 \%$ $(1-\beta=0.20)$. La probabilidad de presencia/ausencia de animal en vivienda, estimada a base de estudios previos en la ciudad de Buenos Aires y partidos del Gran Buenos Aires (Agostini et al., 1986; Cerverizzo et al., 1995; Degregorio et al., 1995; Sommerfelt y Fran- co, 2002) fue de 54 y $46 \%\left(p_{1}=0.54, p_{2}=0.46\right)$ y el error en las estimaciones fue de $10 \%$ (Broll et al., 2002).

Se implementó una encuesta con preguntas semi-estructuradas en relación con las características demográficas de las poblaciones humana y canina, las características de la tenencia de animales (forma de obtención y motivo), las conductas en la tenencia y las motivaciones de las personas en relación con la tenencia (o no) de animales de compañía (caninos). Se solicitó que cada ítem fuera respondido bajo una escala semicuantitativa considerando: 1: no frecuente, 2: poco frecuente, 3 : frecuente, y 4: muy frecuente.

Las diferencias estadísticas entre los dos grupos en estudio se determinaron a través de la prueba de Fisher - para datos cualitativos - y la prueba «t» de Student - para datos cuantitativos.

Se desarrollaron mapas cognitivos para describir las variables definidas. Cada variable fue cuantificada estimando su índice de posición (IP) y su intervalo de $95 \%$ de confianza (Degregorio y Sommerfelt, 2007, 2009). Los mapas cognitivos correspondientes a la tenencia de caninos se elaboraron considerando el IP de la categoría frecuente de cada variable en estudio y el índice de consistencia (IC) del modelo general (Ozesmi U y Ozesmi SL, 2004; Degregorio y Sommerfelt, 2007, 2009). Para evaluar la exactitud de la estimación de mortalidad anual, se comparó el valor obtenido en las encuestas con el cálculo a través de tablas de vida (Di Nardo et al., 2007). Para el análisis estadístico bivariado se empleó la prueba para diferencia de proporciones (Fleiss, 1981).

\section{Resultados}

\section{Estudios Demográficos}

En el Cuadro 1 se presentan los resultados del estudio demográfico en las dos áreas del estudio, determinándose una rela- 
Cuadro 1. Estudio demográfico en viviendas del área San Cristóbal y del área Barrio Mitre, Ciudad Autónoma de Buenos Aires. Argentina, 2013 (expresado en valor absoluto y proporciones)

\begin{tabular}{lcc}
\hline & Grupo 1 - San Cristóbal & Grupo 2 - Barrio Mitre \\
\hline Viviendas encuestadas & 357 & 169 \\
Habitantes & 1373 & 638 \\
Relación Habitante/Vivienda & 2.65 & $3.78^{*}$ \\
Adultos & $827(0.60)$ & $470(0.74)^{* *}$ \\
Menores & $546(0.40)$ & $168(0.26)^{* *}$ \\
\hline
\end{tabular}

$* p<0.01 ; * * p<0.001$

Cuadro 2. Estudios de características de la tenencia de animales, particularmente caninos en viviendas del área San Cristóbal y del área Barrio Mitre, Ciudad Autónoma de Buenos Aires, Argentina. 2013 (expresado en valor absoluto y proporciones)

\begin{tabular}{lcc}
\hline & $\begin{array}{c}\text { Grupo 1 - San } \\
\text { Cristóbal }\end{array}$ & $\begin{array}{c}\text { Grupo 2 - Barrio } \\
\text { Mitre }\end{array}$ \\
\hline Animales/vivienda & $244 / 357(0.68)$ & $108 / 169(0.64) \mathrm{ns}$ \\
Vivienda con canino/con animales & $171 / 244(0.70)$ & $97 / 108(0.90) *$ \\
Relación Canino/Vivienda & $210 / 357(0.59)$ & $180 / 169(1.07) *$ \\
Relación Persona/Canino & $1373 / 210(6.54)$ & $638 / 180(3.54) * *$ \\
Caninos (número) & 210 & 180 \\
Hembra & $123(0.59)$ & $91(0.51) \mathrm{ns}$ \\
Macho & $87(0.41)$ & $89(0.49) \mathrm{ns}$ \\
Raza pura & $93(0.44)$ & $47(0.26) *$ \\
Talla pequeña-mediana & $161(0.77)$ & $143(0.79) \mathrm{ns}$ \\
Mortalidad anual (p (IC)) & $0.11(0.06-0.15)$ & $0.12(0.07-0.17) \mathrm{ns}$ \\
Supervivencia (años \pm de) & $12.2 \pm 2.9$ & $8.5 \pm 2.5 * *$ \\
Natalidad anual (p (IC)) & $0.11(0.05-0.16)$ & $0.28(0.22-0.35) * *$ \\
Esterilización general (p (IC)) & $0.19(0.13-0.25)$ & $0.19(0.13-0.25) \mathrm{ns}$ \\
Hembras esterilizadas (n) & $0.29(0.10-0.37)$ & $0.33(0.23-0.43) \mathrm{ns}$ \\
Machos esteriliza dos (n) & $0.05(0.01-0.08)$ & $0.04(0.01-0.08) \mathrm{ns}$ \\
Edad a la esterilización (años \pm de) & $8.2 \pm 6.8$ & $4.5 \pm 3.5 * *$ \\
\hline
\end{tabular}

ns: diferencias no significativas; ${ }^{*} p<0.01 ;{ }^{* *} p<0.001$

p (IC): proporción (intervalo confianza 95\%) 
ción habitante-vivienda de 2.65 y 3.68 para el grupo 1 y 2 , respectivamente, indicando la presencia de 1.13 más habitantes por vivienda en el grupo 2. Se observaron diferencias significativas entre grupos en la distribución por edad (menores/adultos) de los habitantes (p: 0.01).

\section{Tenencia de Animales}

Los resultados relativos a las características de la tenencia de animales, especialmente caninos, se presentan en el Cuadro 2. Se observaron diferencias en la relación de viviendas con caninos y viviendas con animales $(\mathrm{p}<0.01)$, donde en el grupo 1 hay cerca de un canino cada dos viviendas, en tanto que en el grupo 2 hubo casi un canino por vivienda. Asimismo, en el grupo 1 se encontró cerca del doble de personas por canino (6.54:1) mientras que en el grupo 2 fue bastante menos (3.54:1) $(\mathrm{p}<0.001)$. Además, hubo más canes de raza pura en el grupo 1 (0.44 vs $0.26 ; \mathrm{p}<0.01)$.

No se encontró diferencia significativa entre grupos en relación con el porcentaje de mortalidad anual; sin embargo, se observaron marcadas diferencias en la edad promedio de supervivencia $(\mathrm{p}<0.001)$, donde los canes del el grupo 1 sobreviven cerca de 3.7 años adicionales que los canes del grupo 2 , pero tienen una menor natalidad anual $(0.11$ vs. $0.28, \mathrm{p}<0.001)$ (Cuadro 2).

No se observaron diferencias significativas en los índices de esterilización entre grupos, pero la edad media de esterilización en el grupo 2 fue significativamente menor que en el grupo 1 (Cuadro 2).

\section{Motivaciones y Conductas en la Tenencia}

Los encuestados en el grupo 1 obtuvieron sus canes mayormente como regalo (64\%) o comprándolos (48\%), mientras que en el grupo 2 fue principalmente como regalo $(78 \%)(p<0.01)$. La adopción fue una forma de poca relevancia $(<6 \%)$ en la obtención de las mascotas en ambos grupos. La principal razón de tener el perro en ambos grupos fue porque les gusta, seguido porque son para los niños y por las costumbres familiares; sin embargo, la seguridad fue un motivo de importancia en el grupo $1(\mathrm{p}<0.02)$ (Cuadro 3). Asimismo, una alta proporción de los entrevistados en el grupo 2 indicó que su animal duerme fuera (24\%) y come fuera ( $42 \%$ ) en comparación con las respuestas en el grupo $1(\mathrm{p}<0.01)$ (Cuadro 3).

Entre los que mencionan que sus animales duermen dentro de la casa, una alta proporción del grupo 2 mencionó que lo hacen en su lugar (66\%), en la habitación (55\%) y en las camas $(45 \%)(\mathrm{p}<0.01$; Cuadro 3$)$. En referencia a la alimentación, los encuestados en el grupo 1 proporcionan alimentos balanceados con mayor frecuencia que los del grupo $2(57 \%$ vs $37 \%, \mathrm{p}<0.01)$, de allí que la mayor proporción del grupo 2 les alimente con comida elaborada ( $24 \%$, $\mathrm{p}<0.01$; Cuadro 3).

No se observaron diferencias significativas en la proporción de encuestados que mencionaron que llevan o dejan que su animal orine o defeque fuera de su hogar. Por otro lado, la proporción de agresiones señalada por los encuestados fue significativamente superior en el grupo $1(\mathrm{p}<0.01$; Cuadro 3). No se observaron diferencias entre grupos cuando fueron consultados respecto a si alimenta o alimentaría animales sin dueño.

En el Cuadro 4 se indican los motivos de los encuestados de la no tenencia de animales en el momento de la encuesta. La falta de espacio, falta de tiempo para atenderlos y la muerte del animal fueron los factores con mayor frecuencia en ambos grupos. No obstante, las razones de falta de espacio, tiempo para atenderlos, molestia y factores económicos fueron mayores en el grupo 1 en comparación con el grupo 2. 
Cuadro 3. Motivaciones y conductas en la tenencia de animales de compañía en el área San Cristóbal y en el área Barrio Mitre, Ciudad Autónoma de Buenos Aires. Argentina. 2013 (expresado en valor absoluto y proporciones)

\begin{tabular}{|c|c|c|}
\hline & $\begin{array}{l}\text { Grupo } 1 \text { - San } \\
\text { Cristóbal }\end{array}$ & $\begin{array}{c}\text { Grupo 2- Barrio } \\
\text { Mitre }\end{array}$ \\
\hline Vivienda con canino & 171 & 97 \\
\hline $\begin{array}{l}\text { Forma de obtención }{ }^{1} \\
\text { Compra } \\
\text { Recolecta } \\
\text { Regalo } \\
\text { No determinado }\end{array}$ & $\begin{array}{l}83(0.48) \\
63(0.37) \\
109(0.64) \\
28(0.16)\end{array}$ & $\begin{array}{l}20(0.23) * \\
26(0.27) * \\
76(0.78) \mathrm{ns} \\
11(0.11) \mathrm{ns}\end{array}$ \\
\hline $\begin{array}{l}\text { Motivo para tenerlo }{ }^{1} \\
\text { Costumbre familiar } \\
\text { Le gusta } \\
\text { Por los niños } \\
\text { Seguridad } \\
\text { Soledad } \\
\text { Recomendación médica }\end{array}$ & $\begin{array}{l}93(0.54) \\
143(0.84) \\
98(0.57) \\
53(0.31) \\
6(0.04) \\
1(0.006)\end{array}$ & $\begin{array}{l}38(0.39) \mathrm{ns} \\
82(0.85) \mathrm{ns} \\
54(0.56) \mathrm{ns} \\
9(0.09) * \\
11(0.11) \mathrm{ns} \\
1(0.01) \mathrm{ns}\end{array}$ \\
\hline $\begin{array}{l}\text { ¿Dónde duerme? } \\
\text { Afuera } \\
\text { En la cama } \\
\text { En la habitación } \\
\text { En su lugar } \\
\text { En cualquier lugar de la casa }\end{array}$ & $\begin{array}{l}22(0.13) \\
59(0.35) \\
37(0.22) \\
53(0.31) \\
61(0.36)\end{array}$ & $\begin{array}{l}23(0.24) * \\
44(0.45) * \\
54(0.55) * \\
64(0.66) * \\
15(0.15) n s\end{array}$ \\
\hline $\begin{array}{l}\text { ¿Dónde come? } \\
\text { Afuera } \\
\text { En su lugar } \\
\text { En cualquier lugar de la casa }\end{array}$ & $\begin{array}{c}31(0.18) \\
147(0.86) \\
24(0.14)\end{array}$ & $\begin{array}{l}41(0.42) * \\
84(0.86) \mathrm{ns} \\
10(0.10) \mathrm{ns}\end{array}$ \\
\hline $\begin{array}{l}\text { Alimentación } \\
\text { Prepara comida a veces } \\
\text { Nunca prepara comida } \\
\text { Siempre prepara comida } \\
\text { Come balanceado } \\
\text { Come comida elaborada } \\
\text { Come excedentes } \\
\text { Come de todas las opciones }\end{array}$ & $\begin{array}{l}97(0.57) \\
68(0.39) \\
45(0.26) \\
97(0.57) \\
19(0.11) \\
44(0.26) \\
11(0.06)\end{array}$ & $\begin{array}{l}31(0.32) \mathrm{ns} \\
30(0.31) \mathrm{ns} \\
36(0.37) \mathrm{ns} \\
36(0.37){ }^{*} \\
23(0.24) * \\
22(0.23) \mathrm{ns} \\
13(0.13) \mathrm{ns}\end{array}$ \\
\hline $\begin{array}{l}\text { Orina/defeca } \\
\text { Fuera } \\
\text { Su lugar } \\
\text { Cualquier lugar }\end{array}$ & $\begin{array}{l}157(0.92) \\
96(0.56) \\
75(0.44)\end{array}$ & $\begin{array}{c}83(0.86) \mathrm{ns} \\
59(0.61) \\
38(0.39)\end{array}$ \\
\hline $\begin{array}{l}\text { Vínculos } \\
\text { Salen a la calle } \\
\text { Salen con control directo } \\
\text { Agredió (último año) } \\
\text { Atención veterinaria } \\
\text { At. veterinaria (último año) } \\
\text { Alimenta animales sin dueño }\end{array}$ & $\begin{array}{c}151(0.88) \\
138 / 151(0.91) \\
21(0.12) \\
155(0.91) \\
120 / 155(0.77) \\
52(0.30)\end{array}$ & $\begin{array}{l}76(0.78) \mathrm{ns} \\
61 / 76(0.81) \mathrm{ns} \\
5(0.05)^{*} \\
81(0.84) \mathrm{ns} \\
76 / 81(0.93) \mathrm{ns} \\
36(0.37) \mathrm{ns}\end{array}$ \\
\hline
\end{tabular}

ns: diferencias no significativas; ${ }^{*} p<0.01 ;{ }^{* *} p<0.001$

${ }^{1}$ Variable con respuestas múltiples 
Cuadro 4. Motivos de no tenencia de animales en las áreas San Cristóbal y Barrio Mitre, Ciudad Autónoma de Buenos Aires, Argentina. 2013 (expresado en valor absoluto y proporciones)

\begin{tabular}{lcc}
\hline & $\begin{array}{c}\text { Grupo 1 - San } \\
\text { Cristóbal }\end{array}$ & $\begin{array}{c}\text { Grupo 2 - Barrio } \\
\text { Mitre }\end{array}$ \\
\hline Falta de espacio & $84(0.74)$ & $21(0.34) * *$ \\
Falta tiempo para atender & $65(0.58)$ & $17(0.28) *$ \\
Murió & $30(0.27)$ & $15(0.25) \mathrm{ns}$ \\
Molestan & $26(0.23)$ & $7(0.11)^{*}$ \\
Causas económicas & $26(0.23)$ & $5(0.08)^{*}$ \\
Por recomendación médica & $13(0.12)$ & $5(0.08) \mathrm{ns}$ \\
Conductas de los animales & $16(0.14)$ & $2(0.03) *$ \\
No le permiten & $17(0.15)$ & $1(0.02) *$ \\
\hline
\end{tabular}

ns: diferencias no significativas; ${ }^{*} p<0.01 ;{ }^{* *} p<0.001$

\section{Mapas Cognitivos de Tenencia}

En las figuras 1 y 2 se presentan los análisis de las motivaciones, las actitudes y las conductas de los encuestados en los grupos encuestados (San Cristóbal y Barrio Mitre) en cuanto a la tenencia de caninos. El análisis estadístico de factores relacionados con los motivos desfavorables para la tenencia y la relación de factores económicos, falta de tiempo y de espacio indicaron asociación significativa (grupo 1: $\mathrm{p}<0.01$; grupo 2; $\mathrm{p}<0.05)$. El índice de consistencia del modelo (CI) fue 0.052 para el grupo 1 y 0.067 para el grupo 2 .

\section{Discusión}

La proporción de habitantes por vivienda y la proporción de adultos por vivienda encuestada fue superior en el grupo 2 (Barrio Mitre), superando en aproximadamente el 50\% de los índices medios para la Ciudad de Buenos Aires, a diferencia del grupo 1 (San Cristóbal) que presentó índices muy cerca- nos a los medios de la ciudad. Estos resultados permitirían asegurar la representatividad de los grupos bajo estudio del nivel socioeconómico al cual pertenecen (INDEC, 2001).

La proporción de animales por vivienda en las dos áreas superan las publicadas previamente para la Ciudad de Buenos Aires (Sommerfelt y Franco, 2002) y en sus áreas periféricas (Agostini et al., 1986; Cerverizzo et al., 1995). Sin embargo, el área Barrio Mitre presentó indicadores relacionados con presencia de caninos en viviendas muy superiores al área San Cristóbal. Esta variabilidad observada en los índices de tenencia de caninos podría estar reflejando grandes diferencias en la tenencia de animales directamente relacionadas con el área geográfica en estudio y el nivel socioeconó-mico de sus habitantes (Degregorio, 2010).

En 1990, la Organización Mundial de la Salud (WHO-WSPA, 1990) postuló que la relación promedio ideal es un canino cada diez habitantes. Ese índice, en países en desarro$1 l o$, ha de ser entre 7 y 10 habitantes por cani- 


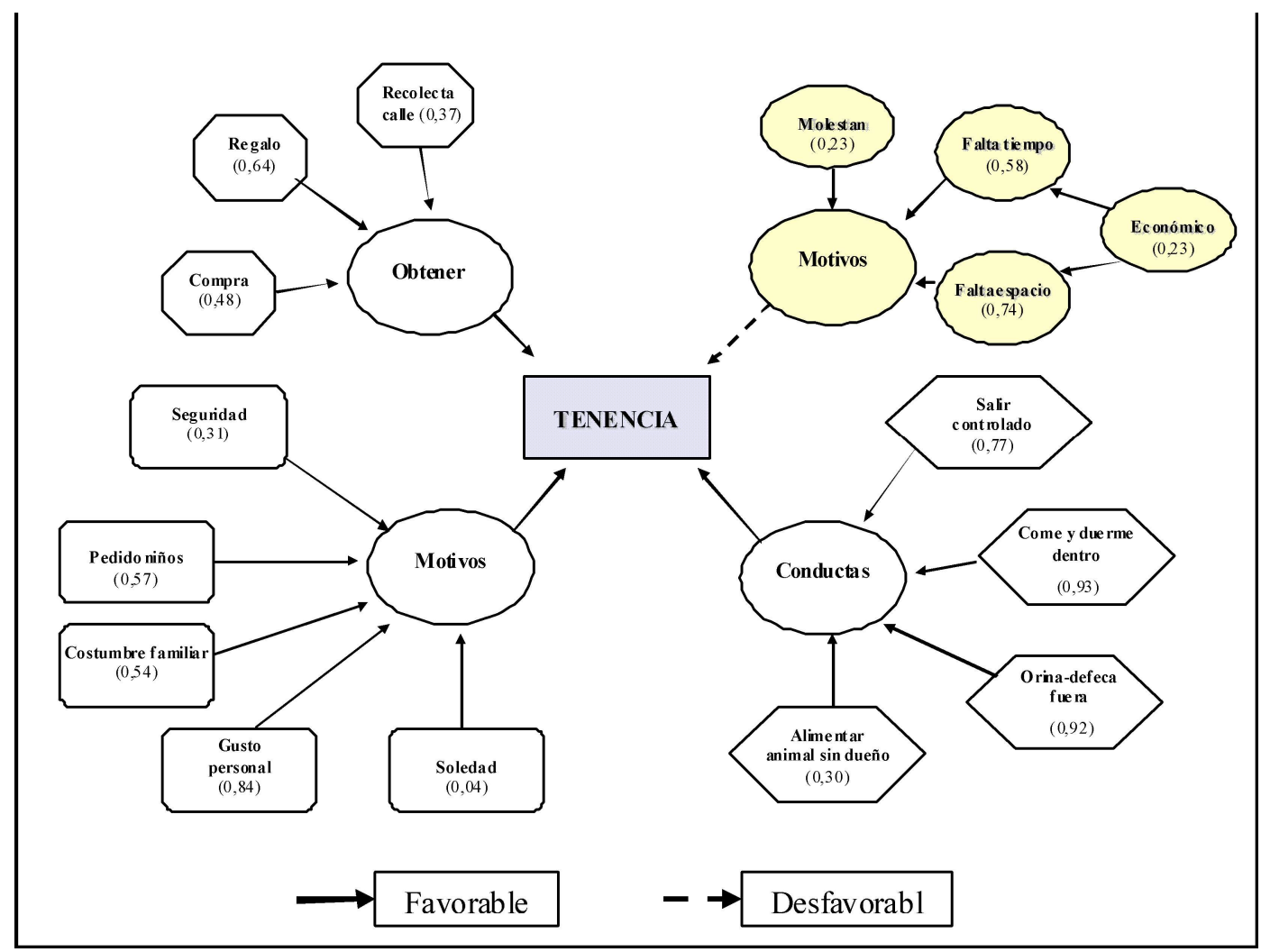

Figura 1. Mapa cognitivo de la tenencia de caninos en encuestados en el área de San Cristóbal, Ciudad Autónoma de Buenos Aires, Argentina. 2013

no en consideración con factores socioeconómicos y de transmisión de enfermedades. En el presente estudio, este índice ha sido inferior en ambas áreas (6.5 en San Cristóbal y 3.5 en Barrio Mitre), indicando el estrecho vínculo entre el hombre y caninos en Buenos Aires, aunque con variaciones de acuerdo al nivel socioeconómico. Si bien se considera que el indicador debería ser ajustado de acuerdo a los nuevos estudios realizados en el país y en otros países de América (Sommerfelt y Franco, 2002; Degregorio et al., 2005; Degregorio y Sommerfelt, 2007, 2009; Acosta Jamett et al., 2010), se debería considerar un mayor análisis del vínculo persona-animal, de acuerdo a las características de tenencia en cada comunidad.

En el estudio de la población canina y su distribución por sexo se observó una mayor proporción de hembras, sin diferencias significativas en ambos grupos. Esto podría indicar una mayor estabilidad en la estructura poblacional canina en la ciudad (Degregorio et al., 1995; Trinidad et al., 1995; Bennett y Rohlf, 2007; Slater et al., 2008). Asimismo, una alta proporción de animales de talla pequeña y mediana en ambas áreas estaría asociado al espacio reducido de las viviendas en la ciudad, en tanto que la mayor frecuencia de razas puras en el área San Cristóbal podría atribuirse a diferencias socioeconómicas entre las áreas en estudio.

Si bien no se observó mayores diferencias en mortalidad anual entre grupos, la supervivencia media fue inferior en Barrio Mitre, aunque superior a tasas en América Latina (Agostini et al., 1986; Ibarra et al., 1991; Acosta Jamett et al., 2010). Por otro lado, la natalidad anual es el doble en el área Barrio Mitre. Esto podría explicarse plantean- 


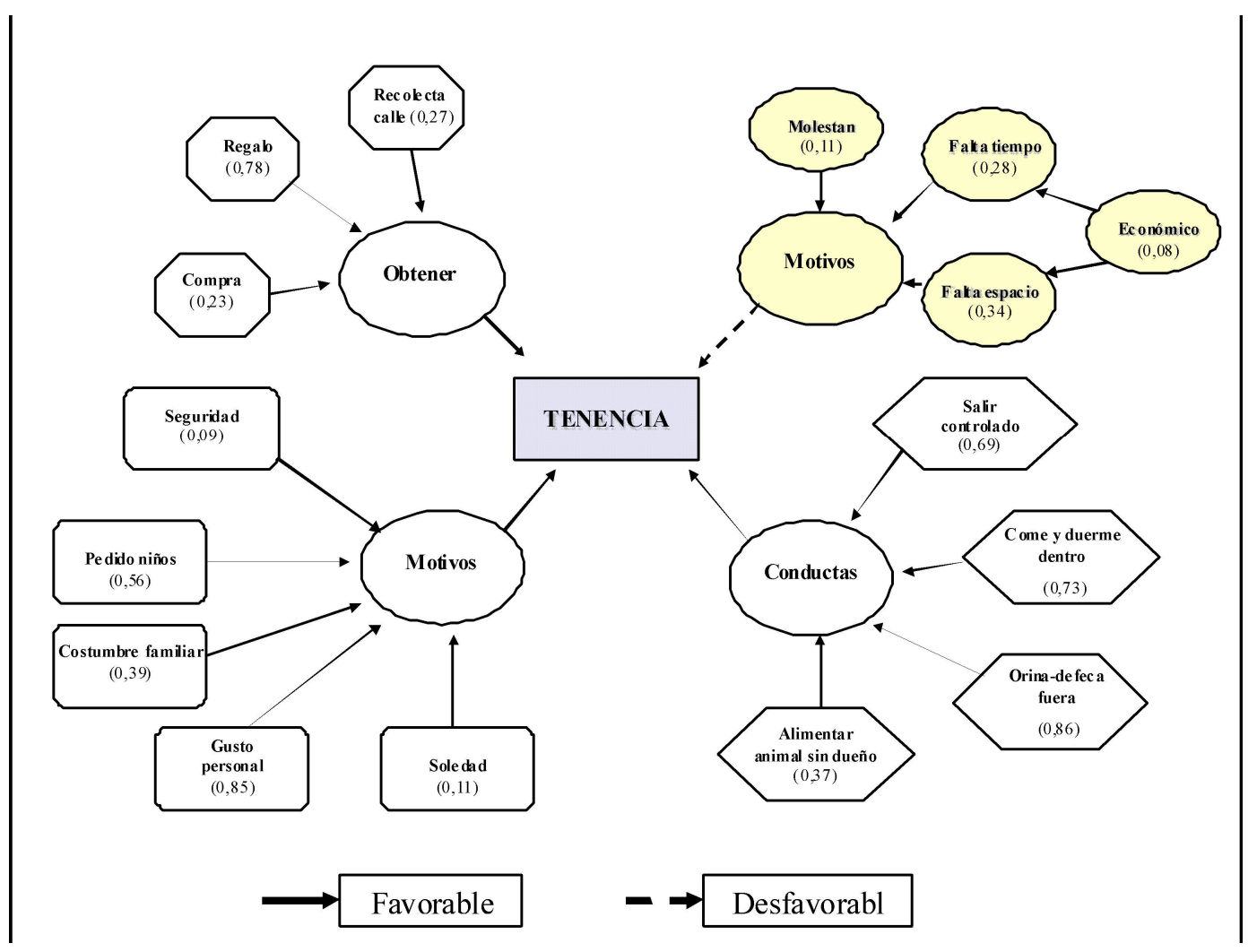

Figura 2. Mapa cognitivo de la tenencia de caninos en el área Barrio Mitre, Ciudad Autónoma de Buenos Aires, Argentina. 2013

do que las poblaciones de animales que reciben mejores cuidados, como en el área San Cristóbal, las tasas de reproducción y mortalidad disminuyen y mejoraría la expectativa de vida (WHO-WSPA, 1990).

Se ha planteado que el mayor impacto para contener el crecimiento de las poblaciones caninas es a través de la esterilización (Di Nardo et al., 2007; Slater et al., 2008). En el presente estudio no se observaron diferencias en la proporción general y por sexo de esterilización quirúrgica entre ambos grupos en estudio, salvo diferencias en la edad media en que fueron esterilizados. Por otro lado, se ha planteado que niveles adecuados de animales esterilizados permitirían el óptimo control del crecimiento de la población animal (Di Nardo et al., 2007; Slater et al., 2008); sin embargo, otros estudios mencionan que aun con altos índices de esteriliza- ción, la tasa de crecimiento continúa siendo positiva (Tortosa et al., 2008; Zumpano et al., 2012).

Si se considera que el grupo que potencialmente requiere mayor control reproductivo es aquel que habita en casas, los canes que viven en departamentos serían una población fácil de controlar con la limitación inherente de sus salidas al espacio público. Los resultados observados podrían indicar que la esterilización quirúrgica es empleada en distintas áreas con diferente estrategia, pues en el área San Cristóbal se emplearía como un método que elegiría el tenedor por comodidad, para evitar características no agradables de la convivencia humano-animal en espacios limitados; en cambio, en el área Barrio Mitre se aplicaría como control poblacional, lo cual explicaría la edad temprana de intervención. 
Se ha planteado que, en el estudio de las poblaciones de animales de compañía, es menester conocer las razones de la tenencia $\mathrm{y}$ analizar los motivos del vínculo humanoanimal (Lord et al., 2007). Los estudios de la conducta animal a través de las motivaciones, las actitudes y las conductas expresadas por el tenedor son un campo en desarrollo que intenta conocer cómo la apreciación subjetiva expresa las conductas del animal y los vínculos con sus tenedores (Kirkden y Pajor, 2006).

En acuerdo con estudios previos (Degregorio, 2010), se observó que, mayoritariamente en ambos grupos, los caninos fueron incorporados a través del regalo, la compra o la recolección en la vía pública. Es particularmente importante señalar el bajo porcentaje de encuestados que consideraron la adopción como una forma válida para obtener su canino. A la vez, ambos grupos en estudio coincidieron que los principales motivos para tener animales son el gusto personal, las costumbres familiares y la solicitud de los niños, mientras que una baja proporción de encuestados en ambos grupos mencionó la soledad como motivo para incorporar un animal al hogar.

En concordancia con otros estudios (Sommerfelt y Franco, 2002) y con independencia de las diferencias entre ambos grupos, se puede considerar que la mayor proporción de animales comen y duermen dentro del hogar, pero orinan y defecan fuera de su hogar, en el espacio público. La tenencia responsable implica derechos y obligaciones, centrados en la adecuada convivencia en el ámbito urbano. Estos resultados estarían indicando que la relación humano-animal, muy frecuentemente, se trasladaría del ámbito privado al espacio público, sobre todo en aspectos «molestos» en la tenencia del animal con el potencial impacto ambiental y riesgo de transmisión de enfermedades (Faulkner, 1985).

Los niveles de atención profesional y las formas de alimentación han sido considerados como indicadores de desarrollo y de ni- vel socioeconómico de las poblaciones (Degregorio, 2010). Si bien en este estudio se observó un alto nivel de atención profesional en ambos grupos, diferencias significativas se observaron en las formas de alimentación. El empleo de alimentos balanceados se expresó con mayor proporción en San Cristóbal, asociado al nivel socioeconómico de esa área. Asimismo, se destacó que cerca de un tercio de los encuestados en ambos grupos respondió en forma afirmativa respecto a alimentar animales sin dueño. Si bien es considerado realizar estudios más exhaustivos en esta temática, es posible considerar dos aspectos contrapuestos que es el vínculo afectivo humano-animal frente al desconocimiento de riesgos sanitarios implícitos en la situación.

El estudio sobre las motivaciones y conductas de las personas que no tienen animales acordaron que la falta de espacio y tiempo y su relación con factores económicos son las principales causas para no tenerlos. Esto coincide parcialmente con lo expresado por Sommerfelt y Franco (2002) en la Ciudad de Buenos Aires, donde más del $60 \%$ de los entrevistados mencionó estos factores como relevantes. No obstante, pocos encuestados, especialmente en el área Barrio Mitre, consideraron que el animal es una molestia como motivo, fortaleciendo la hipótesis que la no tenencia de animales es un evento transitorio en una alta proporción de la población (Degregorio, 2010).

El análisis de los factores relacionados con la tenencia de caninos a través de mapas cognitivos en ambos grupos y sus respectivos índices de consistencia indicaron el peso relativo de las variables en estudio bajo un mismo modelo. Si bien este modelo coincide con otros, cabe destacar que la combinación de variables en el modelo de los tenedores difiere significativamente del desarrollado por expertos en el tema (Degregorio y Sommerfelt, 2010). En el modelo previo se incluyeron actitudes señaladas por los expertos como predictores de conductas; en cambio, en este estudio, se consideraron para la 
construcción del modelo las conductas identificadas fehacientemente en la población estudiada.

\section{Conclusiones}

- En general, se observó una alta proporción de animales por vivienda en ambas áreas en estudio; sin embargo, los índices de tenencia de caninos por habitante, que expresan el estrecho vínculo entre el hombre y caninos, difieren significativamente, lo que puede atribuirse al diferente nivel socioeconómico.

- La mayor proporción de caninos hembra, de talla pequeña y con tendencia a razas puras con indicadores de mortalidad, natalidad y supervivencia variables indicaría que las poblaciones animales tienden a ser reflejo de las condiciones sociales y los cuidados prodigados y en acuerdo a las características de vida de la población humana.

- Hubo un nivel de esterilización similar en ambos grupos pero con diferencias en la edad media del evento, lo que puede reflejar diferentes necesidades, en algunos casos controlando indicadores reproductivos y en otras por necesidades de la estrecha convivencia en el área urbana.

- Los caninos son incorporados a las familias a través del regalo, compra o recolección en la vía pública, donde la adopción no es considerada como opción. Asimismo, los principales motivos para tener animales son el gusto personal, las costumbres familiares, y por solicitud de los niños. Muy pocos mencionaron la soledad como motivo para incorporar un animal al hogar.

- La mayoría de los animales tiene un buen nivel de atención profesional, comen y duermen dentro del hogar, pero una alta proporción orina y defeca en el ámbito público, con el consiguiente impacto en salud ambiental y la potencial transmisión de enfermedades.
- Los mapas cognitivos de tenencia de caninos a base de encuestas a tenedores y su comparación con los desarrollados en grupos de expertos demostraron ser una novedosa forma de analizar la interrelación de variables y, a la vez, caracterizar cualitativa y cuantitativamente el vínculo entre animales y sus tenedores.

\section{Literatura CitAda}

1. Alcock J. 2003. A textbook history of animal behaviour. Anim Behav 65: 3-10. doi: 10.1006/anbe.2002.2044

2. Acosta Jamett G, Cleaveland C, Cunningham AA, Bronsvort BM. 2010. Demography of domestic dogs in rural and urban areas of the Coquimbo region of Chile and implications for disease transmission. Prev Vet Med 94: 272-281. doi: 10.1016/j.prevetmed. 2010.01.002

3. Adams CL, Bonnett BN, Meek AH. 2000. Predictors of owner response to companion animal death in 177 clients from 14 practices in Ontario. $\mathrm{J}$ Am Vet Med Assoc 217: 1303-1309.

4. Agostini A, Franco A, Sommerfelt I, Lema J, Kistermann JC. 1986. Aspectos de la demografía canina y felina en el partido de Gral. San Martin, Bs. As. 1980. Rev Med Vet 67: 32-37.

5. Barberá Heredia E. 1999. Marco conceptual e investigación de la motivación humana. REME 2(1). [Internet]. Disponible en: http://reme.uji.es/articulos/ abarbe127211298/texto.html

6. Bennett PC, Rohlf VI. 2007. Ownercompanion dog interactions: relationships between demographic variables, potentially problematic behaviours, training engagement and shared activities. Appl Anim Behav Sci 102: 65-87. doi: 10.1016/ j.applanim.2006.03.009

7. Blackshaw JK, Day C. 1994. Attitudes of $\operatorname{dog}$ owners to neutering pets: demographic data and effects of owner attitudes. Aust Vet J 71: 113-116. 
8. Broll S, Glaser S, Kreienbrock L. 2002. Calculating sample size bounds for logistic regression. Prev Vet Med 54: 105-111. doi: 10.1016/S0167-5877(02)00012-0

9. Cerverizzo IJ, Degregorio OJ, Eyherabide RE. 1995. Población animal en el Municipio de Gral. San Martín, Provincia de Buenos Aires. II Aspectos sanitarios. Rev Med Vet 76: 305-308.

10. Degregorio OJ, Cerverizzo IJ, Eyherabide RE. 1995. Población animal en el Municipio de Gral. San Martín, Provincia de Buenos Aires. I Aspectos demográficos. Rev Med Vet 76: 255-258.

11. Degregorio OJ, López CM, Sommerfelt IE. 2005. Tenencia de animales de compañía. Rev Med Vet 86: 141-145.

12. Degregorio OJ, Sommerfelt IE. 2007. Percepciones que tienen los médicos veterinarios en relación con la tenencia de animales de compañia en la ciudad de Buenos Aires. Rev Med Vet 88: 144148.

13. Degregorio OJ, Sommerfelt IE. 2009. Percepciones de los médicos veterinarios en relación con la tenencia de felinos en la ciudad de Buenos Aires. Argentina. Rev Inv Vet Perú 20: 221-227. doi: 10.15381/rivep.v20i2.610

14. Degregorio OJ. 2010. Poblaciones de caninos urbanas: modelos epidemiológicos. Tesis Doctoral. Universidad de Buenos Aires, Argentina. $230 \mathrm{p}$.

15. Di Nardo A, Candeloro L, Budke CM, Slater MR. 2007. Modelling the effect of sterilization rate on owned dog population size in central Italy. Prev Vet Med 82: 308-313. doi: 10.1016/ j.prevetmed.2007.06.007

16. Faulkner LC. 1985. Dimensions of the pet population problem. J Am Vet Med Assoc 166: 477-478.

17. Fleiss JL. 1981. Statistical methods for rates and proportions. $2^{\text {nd }} \mathrm{ed}$. New York: J Wiley \& Sons. 389 p.

18. Franco A, Sommerfelt I, Kistermann JC. 1989. Estructura y dinámica de la población canina y del comportamiento en la tenencia de caninos en un área de la Ciudad de Buenos Aires. En: Word Zoonoses Congresses. Porto Alegre, Brasil. [Internet]. Disponible en: http:// www.buenos aires.gov.ar.

19. Gobierno Ciudad de Buenos Aires. 2005a. Encuesta Anual de hogares. [Internet]. Disponible en: http:// www.estadisticaciudad.gob.ar/eyc/ ?page_id $=702$

20. Gobierno Ciudad de Buenos Aires. 2005b. Unidad de Sistemas de Información Geográfica, Gobierno de la Ciudad de Buenos Aires - USIG-CABA (2005). [Internet]. Disponible en: http:// data.buenosaires.gob.ar/dataset/barrios

21. Ibarra LM, Núñez FS, Cisternas LP, Méndez MP. 1991. Demografía canina y felina en la comuna de la granja, Santiago, Chile. Av Cienc Vet 6: 141-145.

22. [INDEC] Instituto Nacional de Estadísticas y Censos. 2001. Censo nacional de población y vivienda 2001. República Argentina. [Internet] http:// www.indec.mecon.ar/

23. Kirkden RD, Pajor EA. 2006. Using preferences, motivation and aversion tests to ask scientific questions about animals' feelings. Appl Anim Behav Sci 100: 29-47. doi: 10.1016/j.applanim. 2006.04.009

24. Lord LK, Wittum TE, Scarlett JM. 2007. Use of group-randomized trials in pet population research. Prev Vet Med 82: $167-175$.

25. Martínez MC, Tortosa A, Molina JL, Pisapía G, Marcos EM, Degregorio OJ. 2013. Evaluación de sistemas de salud animal: demanda de servicios en la ciudad Autónoma de Buenos Aires. Rev Inv Vet Perú 24: 503-509. doi: 10.15381/rivep.v24i4.2743

26. Ozesmi U, Ozesmi SL. 2004. Ecological models based on people's knowledge: a multi-step fuzzy cognitive mapping. Ecol Model 176: 43-64. doi: 10.1016/j.ecolmodel.2003.10.027

27. Slater MR, Di Nardo A, Pediconi O, Dalla Villa $R$, Candelorio $L$, Alessandrini B, Del Papa S. 2008. Cat and $\operatorname{dog}$ ownership and management 
patterns in central Italy. Prev Vet Med 85: 267-294. doi: 10.1016/j.prevetmed. 2008.02.001

28. Sommerfelt IE, Franco AJ. 2002. Relaciones entre el hombre y los animales de compañía. Rev Med Vet 83: 181-184.

29. Teclaw R, Mendlein J, Garbe P, Mariolis P. 1992. Characteristics of pet populations and households in the Purdue comparative Oncology Program catchments area, 1988. J Am Vet Assoc 201: 1725-1729.

30. Tortosa A, Zumpano R, Degregorio OJ. 2008. Estudio preliminar del impacto de la esterilización en el índice de crecimiento de la población de caninos. En:
III Congreso Latinoamericano de Zoonosis. Buenos Aires, Argentina.

31. Trinidad JJ, Sommerfelt I, Cornero F. 1995. Demografía canina en un partido del Gran Buenos Aires. Argentina. Rev Med Vet 76: 202-204.

32. WHO-WSPA. 1990. Guidelines for dog population management. Ginebra: WHO-WSPA. $165 \mathrm{p}$.

33. Zumpano R, Tortosa A, Degregorio OJ. 2012. Estimación del impacto de la esterilización en el índice de crecimiento de la población de caninos. Rev Inv Vet Perú 22: 336-341. doi: 10.15381/ rivep.v22i4.333 